\title{
A Methodology for Validating Artifact Removal Techniques for Physiological Signals
}

\author{
Kevin T. Sweeney, Student Member, IEEE, Hasan Ayaz, Member, IEEE, Tomás E. Ward, Senior Member, IEEE, \\ Meltem Izzetoglu, Seán F. McLoone, Senior Member, IEEE, and Banu Onaral, Fellow, IEEE
}

\begin{abstract}
Artifact removal from physiological signals is an essential component of the biosignal processing pipeline. The need for powerful and robust methods for this process has become particularly acute as healthcare technology deployment undergoes transition from the current hospital-centric setting toward a wearable and ubiquitous monitoring environment. Currently, determining the relative efficacy and performance of the multiple artifact removal techniques available on real world data can be problematic, due to incomplete information on the uncorrupted desired signal. The majority of techniques are presently evaluated using simulated data, and therefore, the quality of the conclusions is contingent on the fidelity of the model used. Consequently, in the biomedical signal processing community, there is considerable focus on the generation and validation of appropriate signal models for use in artifact suppression. Most approaches rely on mathematical models which capture suitable approximations to the signal dynamics or underlying physiology and, therefore, introduce some uncertainty to subsequent predictions of algorithm performance. This paper describes a more empirical approach to the modeling of the desired signal that we demonstrate for functional brain monitoring tasks which allows for the procurement of a "ground truth" signal which is highly correlated to a true desired signal that has been contaminated with artifacts. The availability of this "ground truth," together with the corrupted signal, can then aid in determining the efficacy of selected artifact removal techniques. A number of commonly implemented artifact removal techniques were evaluated using the described methodology to validate the proposed novel test platform.
\end{abstract}

Index Terms-Artifact removal, electroencephalography (EEG), functional Near-Infrared Spectroscopy (fNIRS), recording methodology.

\section{INTRODUCTION}

$\mathbf{M}$ EASUREMENT of the numerous physiological signals of the human brain has, for a long time, been a critical step in determining a subject's well-being. Functional brain

Manuscript received February 15, 2012; revised April 24, 2012; accepted June 22, 2012. Date of publication July 10, 2012; date of current version September 20, 2012. This work was supported in part by the Irish Research Council for Engineering, Science and Technology (IRCSET) and Science Foundation Ireland under Research Frontiers Program 2009 and under Grant 09/RFP/ECE2376.

K. T. Sweeney, T. E. Ward, and S. F. McLoone are with the Department of Electronic Engineering, National University of Ireland, Maynooth, Ireland (e-mail: ksweeney@eeng.nuim.ie; tomas.ward@eeng.nuim.ie; sean.mcloone@ eeng.nuim.ie).

H. Ayaz, M. Izzetoglu and B. Onaral are with the School of Biomedical Engineering, Science and Health Systems, Drexel University, Philadelphia, PA 19104 USA (e-mail: hasan.ayaz@drexel.edu; meltem@cbis.ece.drexel.edu; banu.onaral@drexel.edu).

Color versions of one or more of the figures in this paper are available online at http://ieeexplore.ieee.org.

Digital Object Identifier 10.1109/TITB.2012.2207400 monitoring is an increasingly important aspect of patient monitoring especially outside the clinical environment. These measures have utility in tracking the deterioration of mental function in conditions such as Alzheimer's disease and dementia as well as for more acute event capture, for instance, with epilepsy. Electroencephalography (EEG) and functional near-infrared spectroscopy (fNIRS) are two noninvasive and relatively portable techniques commonly employed to record physiological signals associated with brain function. These recordings can, however, be highly susceptible to artifacts originating from the subject's motion, as well as other external noise sources.

EEG uses electrodes to record the voltage fluctuations at the scalp due to changing electrical neuronal activity within the brain. Although the amplitude of the electrical potential produced by a single neuron within the cortex is too small to be detected noninvasively, EEG can detect the combination of an ensemble of a large number of neurons. Hence, although EEG has very good temporal resolution (in the order of milliseconds), the spatial resolution is poor with respect to other recording modalities.

fNIRS is an increasingly popular technique used to monitor cerebral hemodynamic changes during brain activity. The regional brain activation causes a change in both oxygenatedhaemoglobin (oxy-Hb) and deoxygenated-haemoglobin (deoxy-Hb) concentration levels local to the active area. As haemoglobin is the main chromophore in the near-infrared light range (between 690 and $900 \mathrm{~nm}$ ), fNIRS uses light at multiple wavelengths in this range to penetrate the skull and assesses the change in oxy- $\mathrm{Hb}$ and deoxy- $\mathrm{Hb}$ concentrations through the change in light absorption [1]. As fNIRS uses optical techniques to record the changes in cortical activation, it is considerably less susceptible to artifacts from electrical sources, both physiological (e.g., eye blinks, muscle movements) and external (e.g., instrumentation noise).

One class of artifacts that can have a large detrimental effect on both modalities are motion artifacts. These artifacts can be due to either the movement of the subject or the resulting movement of the recording electrodes/optodes with respect to the skin. These artifacts are of particular concern in ambulatory recording systems [2] or when recording during epileptic activity. EEG is less sensitive than fNIRS to movement of the subjects head; however, this movement can cause an increase in the number of artifacts due to the activation of skeletal muscles. Movements of the EEG electrodes with respect to the scalp can also cause large errors in the recorded signal. These artifacts can be reduced in number and affected by securing both the electrodes and the wiring correctly and by restricting the 
movement of the subject; however, they are difficult to remove completely.

In fNIRS measurements, subject motion can cause blood pooling in the cortical area of interest. This increase/decrease in oxy-Hb/deoxy-Hb levels may mask changes in the true activation level and is thus deemed an artifact. Furthermore, subject motion can indirectly cause movement of the fNIRS optodes and can cause a sudden change in recorded light intensity. When this movement occurs, the coupling between the optode and the skin is often compromised. The resulting artifact is due to the recording of unwanted external light, the reflection of the incident light from the outer skin layer or changing path length.

A number of signal processing algorithms have been previously employed to remove motion artifacts from physiological signals [3]. One dilemma faced by all researchers, when trying to remove motion artifact from the desired signal, is the lack of knowledge of the true form of the original (noise-free) signal. Without knowledge of this signal, it is not possible to accurately determine the efficacy of a given artifact removal technique. It also proves to be difficult to perform a valid comparison of different methods. Currently, comparisons among techniques are achieved using simulated data.

We propose a novel recording methodology for physiological signals which permits the recording of two separate but highly correlated channels. This methodology allows for the recording of a noise-contaminated and a noise-free signal concurrently. By doing so, a "ground truth" signal becomes available to researchers to test the efficacy of their motion artifact removal techniques. The described methodology also incorporates accelerometers to determine the time and magnitude of the induced motion. This information is used for illustration purposes as well as for a reference signal as required in certain artifact removal techniques such as the adaptive filter technique, described in Section III-A.

This paper builds on the preliminary work proposed in [4] by expanding the methodology to include the more widely used EEG modality as well as the previously described fNIRS data. Motion corrupts EEG data in a considerably different manner to fNIRS and, therefore, warrants additional investigation. In addition, to provide a more comprehensive assessment of the efficacy of the approach, results are presented for an experimental study involving a larger cohort of subjects and an expanded set of artifact removal techniques. The measurements collected with the methodology described in this paper, for both fNIRS and EEG, suggest that the configuration effectively provides both a motion corrupted signal and a strongly correlated artifact free equivalent. Consequently, the methodology allows for the acquisition of signal sets ideally suited for the design, test, and validation of motion artifact removal methods.

The utility of the methodology in artifact removal applications is demonstrated on an example set of motion artifact removal techniques selected from the wide range of algorithms that have previously been proposed in the EEG and fNIRS literature [3]. The selected motion artifact removal techniques, namely, adaptive filtering, Kalman filtering, and ensemble empirical mode decomposition with independent component analysis (EEMDICA) are applied to the EEG and fNIRS data collected using

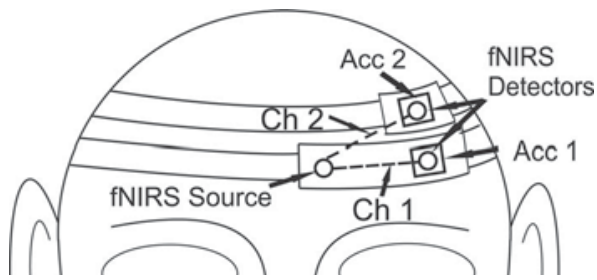

Fig. 1. Position of the fNIRS optodes and accelerometers on the left prefrontal cortex during experiment. Channel 1: positions are fixed. Channel 2: channel path perturbed due to movement of the detector connected to Accelerometer 2.

the proposed system. The methods are representative of the field and range in sophistication from the relatively simple and well known (adaptive filtering) to the more complex and recently proposed (EEMD-ICA). The results from all techniques suggest that the noise within the recordings can be adequately removed, as demonstrated by an increase in signal-to-noise ratio (SNR) and cross-correlation coefficient obtained through the use of the "ground truth" recording provided by the proposed data collection procedure.

The remainder of this paper is structured as follows. Section II describes the novel recording methodology including the recording systems employed and the experimental setup and procedure used. This section also presents example recordings of noise-free and noisy fNIRS and EEG recordings. Section III demonstrates the utility of the methodology using various artifact removal techniques. Finally, Section IV discusses the findings of this paper and future work.

\section{EXPERIMENTAL SETUP}

To facilitate repeatability, this section outlines the systems utilized to record the fNIRS, EEG, and motion data. Furthermore, it describes the setup and positioning of the optodes, electrodes, and accelerometers as well as the general protocol adhered to during experimentation.

\section{A. Data Acquisition System}

The fNIRS data are collected using a TechEn CW6 system (TechEn Inc., MA) at a sampling rate of $25 \mathrm{~Hz}$. This system employs light sources, at wavelengths of 690 and $830 \mathrm{~nm}$, to determine the change in both oxygenated and deoxygenated haemoglobin in the blood as described in Section I. The EEG is recorded using a BioSemi Active-Two system (BioSemi Inc., The Netherlands). DC coupled data are recorded at $2048 \mathrm{~Hz}$. The experiment also utilizes two triaxial accelerometers (ADXL327, Analog Devices) to measure the time, magnitude, and direction of the induced motion. These accelerometers have a full scale range of $\pm 2 \mathrm{~g}$ and are sampled at a frequency of $200 \mathrm{~Hz}$.

1) fNIRS Experimental Setup: The fNIRS light source is connected to a plastic housing which is secured to the forehead of the user using the lower velcro head strap as shown in Fig. 1. The plastic housing is comprised of low-density polythene backed by polyurethane foam. This housing allows for a rigid and secure connection between the fNIRS optode and the head. The lower head strap, which encompasses the source 
optode, also accommodates one of the two detector optodes utilized in the experiment. The spacing between the two optodes is set to $30 \mathrm{~mm}$ conforming with common practice. The second detector is housed on a separate strapping which is not coupled to the first, saving the coupling through the head itself. This detector is strategically positioned above the first as shown in Fig. 1 so as to preserve the $30-\mathrm{mm}$ interoptode spacing between it and the source optode. Moreover, the spacing between the detectors is kept very small (approximately $20 \mathrm{~mm}$ ) so that the overall monitored volume within the head highly overlaps between these two channels. The distance between the centroid points of each source-detector pair is approximately $10 \mathrm{~mm}$.

The two accelerometers utilized during the experiment are employed to determine a measure of differential movement of the detector optodes. To accomplish this, one accelerometer is placed securely onto each individual detector as shown in Fig. 1. Care is taken to ensure that the orientation of each individual accelerometer is kept consistent with respect to the other. It should be again noted that the two detectors and accelerometers are not directly coupled, and therefore, the movement of one will have little or no effect on the position of the second.

2) EEG Experimental Setup: The setup of the EEG experiment is similar to that of the fNIRS experiment. The EEG $(\mathrm{Ag}-\mathrm{AgCl})$ active electrodes, used to record the two separate channels, are secured to the scalp of the subject using a 256 array electrode cap (Electro-cap International, Inc., OH). Electrode gel is placed below each electrode to aid in the coupling with the scalp. The cap is manufactured using a fabric material allowing for the movement of a single electrode without altering the position of the adjacent electrode. The spacing of the recording electrodes is fixed by the cap at $20 \mathrm{~mm}$ allowing for the two channels to be recorded in close proximity. Similar to the fNIRS experiment, the two accelerometers are attached to the individual electrodes, again ensuring that the orientation of each individual accelerometer is kept consistent with respect to the other. In both EEG and fNIRS experiments, all post processing was performed offline using MATLAB (2008b, The MathWorks, Natwick, MA).

\section{B. Experimental Procedure}

Recording of the two modalities was performed independently.

1) fNIRS Experimental Protocol: fNIRS measurements were obtained from ten healthy subjects in order to demonstrate the validity of the proposed methodology. The cohort comprised of four males and six females (mean age: 29 years, standard deviation: 5.62 years). Each recording resulted in two useable trials for each channel due to recording at the two wavelengths (690 and $830 \mathrm{~nm}$ ).

The fNIRS optodes were positioned on the left prefrontal cortex of each subject, as illustrated by Fig. 1. This particular optode positioning was chosen to reduce the possibility of contamination of the signal due to the subjects hair interfering with the coupling between the optode and the skin. Furthermore, as the methodology proposed in this paper is designed to allow for the recording of two highly correlated signals, one with and
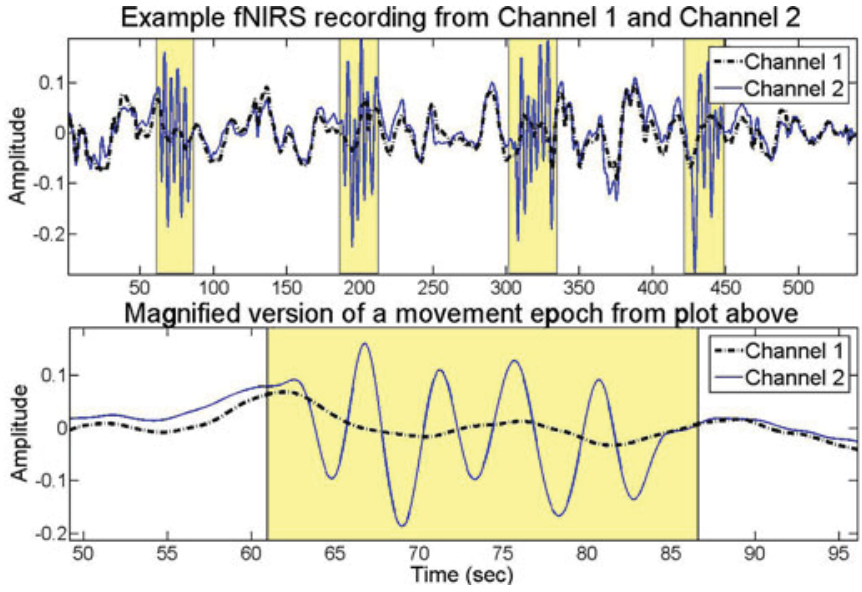

Fig. 2. Example output from an fNIRS recording. The shaded sections highlight the regions containing motion artifact as determined using the accelerometer data. Average correlation over clean epochs: 0.87. Average correlation over movement epochs: 0.31 .

the second without motion artifact contamination, the underlying cortical activity was not of significance. Therefore, the subjects were not required to perform any specific activity for the duration of the experiment.

Due to the setup of the new methodology, the two channels were recorded in close proximity. As the fNIRS optode measures from the volumetric region in between the light source and detector [5], by using the same light source and two separate detectors in close proximity, the optode measurement volumes overlap and the measured cortical activities are expected to be highly correlated for the two optodes. Each individual trial of the experimental session lasted a total of $9 \mathrm{~min}$. At regular 2-min intervals, the experimenter induced a positional disturbance to the detector attached to Accelerometer 2 for a random duration of between 10 and $25 \mathrm{~s}$. This source detector pairing was labeled Channel 2 (see Fig. 1). This slight disturbance, which was performed manually by pulling on the optode cable, induced motion artifact on Channel 2. However, as the detector optode, connected to Accelerometer 1, and the source optode were not disturbed, Channel 1 remained free of contamination throughout the duration of the experiment. This result can be observed in Fig. 2.

The sections of the signal which are not shaded are epochs of motion free data where Channel 2 can be observed to follow the output of Channel 1 closely. These epochs of corrupted data were determined using a simple tagging algorithm [6] using the available data from the accelerometers.

Before each individual recorded trial was selected for use in the analysis performed in this paper, the artifact contaminated signal was analyzed against its corresponding "ground truth" signal. Signals whose clean epochs had an average correlation lower than 0.65 (calculated using the Pearson productmoment correlation coefficient) with the corresponding epoch of the "ground truth" signal were discarded. This ensured that only the most correlated signal pairs were used. Low correlation values during the clean epochs could be due to many factors including poor connection between the optode and the skin. This resulted in 16 fNIRS trials for analysis. These 16 trials were 
Example epoch from EEG recording for Channel 1 and Channel 2
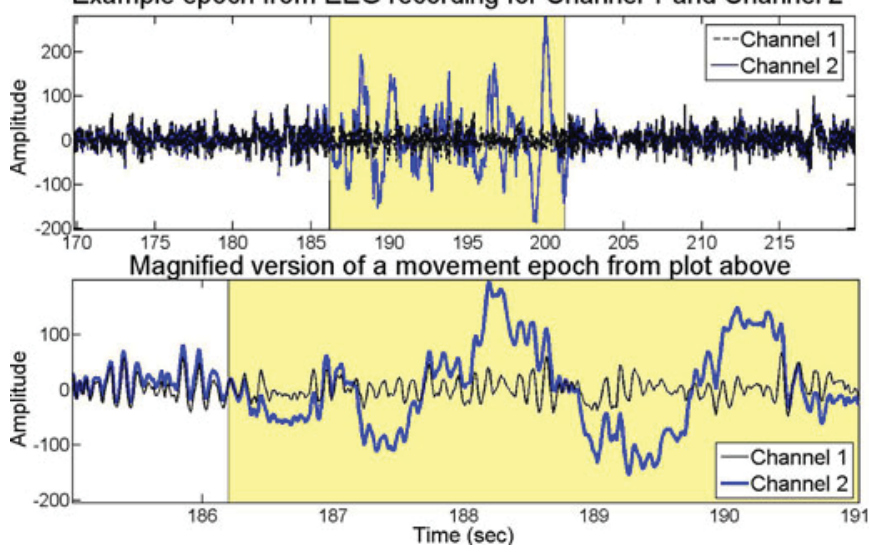

Fig. 3. Example output from an EEG recording. The shaded sections highlight the regions containing motion artifact as determined using the accelerometer data. Average correlation over clean epochs: 0.83. Average correlation over movement epochs: 0.09 .

calculated to have an average correlation $r$ of $0.77(\operatorname{std}=0.2)$ during motion free epochs over all trials. However, the average correlation over the full signal for all trials is significantly lower ( $r=0.58 \mathrm{std}=0.16)$ due to the intermittent presence of motion artifacts. Over all trials, motion artifacts are present in $19.75 \%$ of the data.

2) EEG Experimental Protocol: EEG recordings were obtained, over four sessions, from six healthy subjects (mean age: 27 years, standard deviation: 4.29 years) resulting in 24 trials. The cohort consisted of three males and three females. Two channels of EEG were recorded from the frontal cortex from positions FPz and FP1h (using the 10-5 system as described in [7]), labeled Channel 1 and Channel 2 respectively. The Driven Right Leg and Common Mode Source electrodes were placed at positions P2 and P1, respectively, using the 10-20 system.

Subjects were not required to perform any activity during recording. Each subject was instructed to keep their eyes closed and to maintain a stationary head position throughout the experiment; therefore, limiting the number of artifacts originating from sources such as eye blinks, saccades, and head motion. Again, each trial lasted a total of 9 min with motion induced to Channel 2's electrode at regular 2-min intervals. This motion artifact was induced by mechanically disturbing the electrode by pulling on the connecting lead. An example output from the two channels can be observed in Fig. 3.

During epochs of no motion, the two recording channels were calculated to have an average correlation coefficient $r$ of 0.83 $(\operatorname{std}=0.2)$ but this value again drops $(r=0.40$, std $=0.19)$ when analyzing the full signal due to the presence of artifacts. The artifact components are present in $15.74 \%$ of the data.

\section{VALIDATION OF TEChNiQUES}

In this section, we have illustrated the use of a number of currently available artifact removal techniques to demonstrate the validity of the proposed recording methodology.

The experimental setup for the fNIRS and the EEG, described in Section II, allows for the recording of two separate fNIRS and EEG channels, one clean and the other intermittently contaminated with motion artifact. When motion artifact is induced in one channel, as described in Section II-B1 and II-B2, the two signals can differ greatly depending on the magnitude of the artifact (see Figs. 2 and 3). A comparison of the recordings from Channel 1 and 2, for both recording modalities, was produced based on correlation analysis and SNR. The SNR [measured in decibel $(\mathrm{dB})]$ in this study was calculated using the following formula:

$$
\mathrm{SNR}=10 \log _{10}\left(\frac{\sigma_{x}^{2}}{\sigma_{e}^{2}}\right)
$$

where $\sigma_{x}^{2}$ is the variance of the clean noise-free signal (Channel 1) and $\sigma_{e}^{2}$ is the variance of the error signal. Before the application of the artifact removal technique, the error signal is equal to the motion artifact found by subtracting the noise-free (Channel 1) recording from the noisy (Channel 2) measurement. The error signal after the application of a motion artifact removal technique is the signal found by subtracting the estimated desired signal, found as the outcome of the algorithm used, from the true noise-free signal (Channel 1). During the clean epochs, the signals show a high average SNR (1.93 dB for fNIRS and $5.92 \mathrm{~dB}$ for EEG). This SNR drops considerably when calculated over the full signal $(-3.65 \mathrm{~dB}$ for fNIRS and $-6.13 \mathrm{~dB}$ for EEG). The purpose of the numerous available artifact removal techniques is to increase the SNR of the contaminated signal, while also increasing the correlation of the estimated signal with the (previously unknown) known true signal. Using this new proposed recording methodology, it is now possible to incorporate the previously unavailable "ground truth" signal to aid in the validation of these artifact removal techniques. As the clean signal is used for comparison only, the artifact removal techniques employed must be capable of operating on single-channel measurements. Such a constraint is increasingly common where attempts to improve patient compliance when using telehealth systems have necessitated the use of a minimal instrumentation paradigm [8].

\section{A. Adaptive Filter}

The adaptive filter has been used widely for artifact removal in signal processing for the past number of decades. Applications include the removal of EMG artifacts and ocular artifacts from EEG and motion artifact and physiological signals from fNIRS [3].

The adaptive filter technique operates under the assumption that the desired true signal $s(n)$ and the contaminating artifact $v(n)$ are uncorrelated, i.e.

$$
E[s(n) v(n)]=0
$$

where $E[$.$] is the expectation operation. A second assumption$ made when utilizing the adaptive filter technique is that the true desired signal and the artifact signal are linearly mixed, as shown in Fig. 4. Using a tapped delay line, the filter generates a signal $\hat{v}(n)$, which is correlated with the actual artifact signal $v(n)$, by use of a reference signal $u(n)$. This estimate of the artifact is then subtracted from the recorded signal $x(n)$, and the residual 


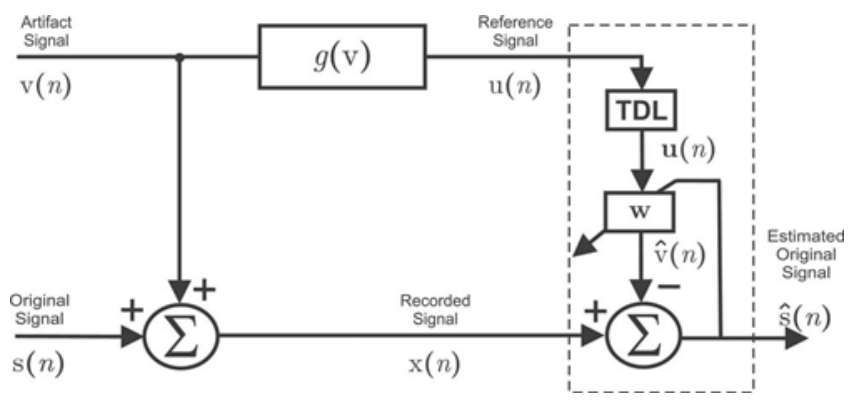

Fig. 4. Adaptive filter framework for artifact removal.
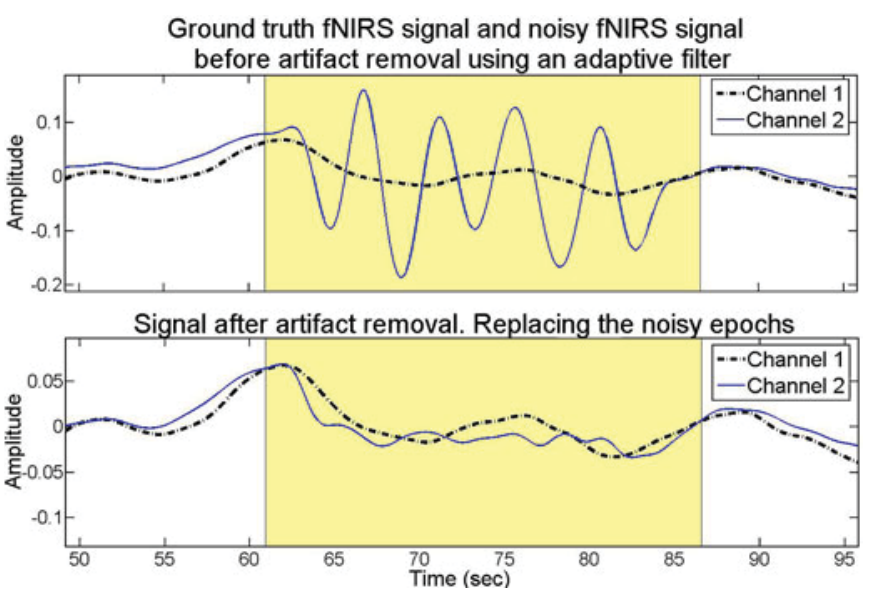

Fig. 5. Example of the output of the adaptive filter using the fNIRS data.

$\hat{s}(n)$ is an estimate of the original signal $s(n)$ [9], i.e.

$$
\hat{s}(n)=x(n)-\hat{v}(n) .
$$

The filter coefficients are adjusted so as to minimize the meansquared amplitude of the estimated signal $\hat{s}(n)$ as this has the effect of suppressing the artifact component but not the desired signal.

The adaptive filter can be easily adopted for usage with the two applications detailed in this paper. The two accelerometers, attached to the optodes or the electrodes, are used to determine when differential movement has occurred in the experiment. When this differential movement does occur, due to the actions of the experimenter, artifacts are observed on one of the independent channels. This differential acceleration, as well as the calculated differential velocity and position, are then used to generate a nonlinear model of the artifact signal. This model is then employed as the reference $u(n)$ for the adaptive filter. The sampling frequencies of all datasets (EEG, fNIRS, and accelerometers) are adjusted to ensure that they are operating on the same sampling frequency prior to implementing the adaptive filter. When using an adaptive filter, the choice of algorithm is of importance, as it dictates the computational cost and accuracy of the filter. For this paper, a normalized least mean square algorithm was implemented [10].

For example, output of the adaptive filter technique plotted against the original noisy signal and the "ground truth" signal can be observed in Figs. 5 and 6 for fNIRS and EEG, respectively. From these figures, it is possible to visually acknowledge
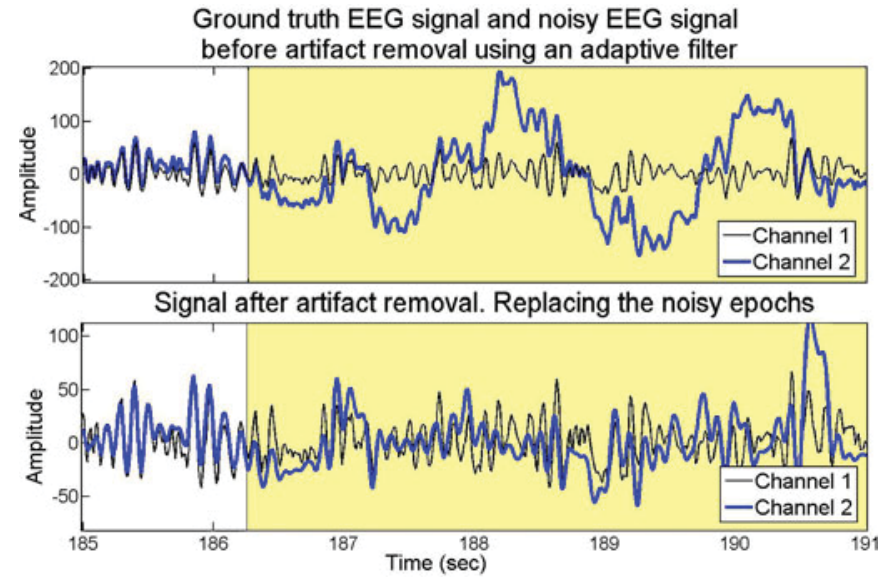

Fig. 6. Example of the output of the adaptive filter using the EEG data.

that the cleaned signal does indeed tend toward the "ground truth" signal generated using the described modality. For the purpose of this paper, only the motion artifact contaminated sections of the signal were replaced with the cleaned section of the signal, and the epochs of clean data were left untouched.

The adaptive filter was run for all trials, on both modalities, to ascertain the change in SNR and correlation values post processing. After running the technique the correlation of Channel 2 with Channel 1 increased by $68.22 \%$ to 0.71 for the fNIRS data and by $37.66 \%$ to 0.56 for the EEG data. This percent correlation increase is calculated as

$$
\frac{R_{\text {after }}-R_{\text {before }}}{R_{\text {clean }}-R_{\text {before }}} * 100 \% \text {. }
$$

$R_{\text {before }}$ is the correlation calculated over the epochs of noisy data as shown by the shaded areas of Figs. 2 and 3. $R_{\text {after }}$ is the correlation over the same epochs after the artifact removal techniques have been employed and $R_{\text {clean }}$ is the correlation over the epochs of known clean data (the nonshaded areas of Figs. 2 and 3).

The SNR also rose for both modalities, by $5 \mathrm{~dB}$ for the fNIRS data and by $5.1 \mathrm{~dB}$ for the EEG data. As both the correlation and the SNR of the signal increased after employing the adaptive filter, it can be confirmed that the filter does indeed aid in the removal of artifacts from the signal. Without the new methodology, it would not be feasible to estimate these values for correlation and SNR.

\section{B. Kalman Filter}

The Kalman filter has been used by a number of researchers for the removal of artifacts; for example, in 2010, Izzetoglu et al. [11] used the Kalman filter to remove motion artifacts from fNIRS recordings while Sameni et al. [12] used the filter to remove environmental and muscle artifacts from ECG signals.

The Kalman filter implements an approximation of the Bayes filter technique. Bayesian filters probabilistically estimate a given systems state from noisy observations. These filters operate using a predictor-corrector methodology. The predictor stage comprises of a time-update model and is used to predict the systems next state given the current state. The corrector stage uses 

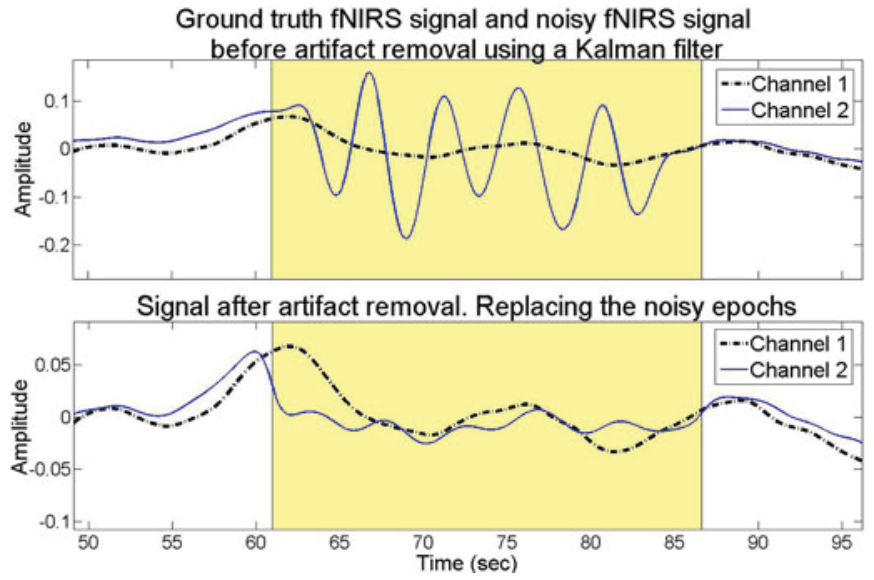

Fig. 7. Example of the output of the Kalman filter using the fNIRS data.

a separate measurement model to correct the predictor estimate given observations of the data. The Kalman filter also incorporates a dynamic gain factor, in the time-update equations, which is automatically updated to specify how much "trust" to put into the observed data. Therefore, if the observations are deemed to be very noisy, more "trust" is placed on the predictor stage, and thus the corrector stage has little impact on the signal. The time and measurement update equations are detailed by Welch and Bishop [13].

Unlike the adaptive filter, a Kalman filter does not require the use of a reference signal to be capable of removing the contaminating artifact. However, the time update model and the measurement model are required a priori and can be determined using a number of different modeling methods such as autoregressive (AR) and moving average.

In this paper, the noise-free fNIRS and EEG data were modeled using an AR model of orders 4 and 5, respectively, using the "ground truth" data from Channel 1. Model orders were determined using the "System Identification Toolbox" from MATLAB. The model parameters were estimated from the available true clean data using the Yule-Walker method. The variance of the measurement noise, required when employing the Kalman filter, was computed using the difference between the noisy signal and the "ground truth" signal. The process noise was estimated using the AR model parameters and the variance of the epoch of clean data, similar to [11]. Example outputs of the Kalman filter for fNIRS and EEG are shown in Figs. 7 and 8, respectively.

Once the parameters of the system were determined, the Kalman filter was applied to all the trials of both the fNIRS and EEG data. After running the technique, the average correlation of noise removed Channel 2 recordings with Channel 1 measurements increased $66.5 \%$ to 0.71 for the fNIRS data and by $83.1 \%$ to 0.76 for the EEG data. The SNR also rose for both modalities, by $5.46 \mathrm{~dB}$ for the fNIRS data and by $9.7 \mathrm{~dB}$ for the EEG data.

\section{Ensemble Empirical Mode Decomposition-ICA}

ICA is a blind source separation (BSS) technique in which recorded, multichannel, signals are separated into their indepen-

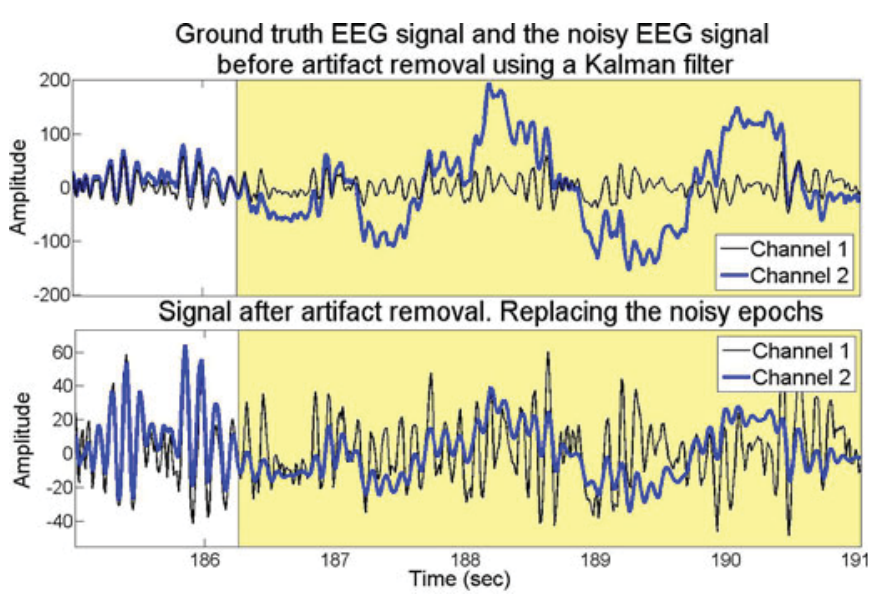

Fig. 8. Example of the output of the Kalman filter using the EEG data.

dent components or sources [14]. BSS is based on a wide class of unsupervised learning algorithms with the goal of estimating sources (which are not necessarily independent) and parameters of a mixing system [15]. ICA operates under a number of assumptions, described by James and Hesse [16], including linear mixing, square mixing, and stationary mixing. Using these assumptions, the ICA algorithms determine the unmixing matrix $\mathbf{W}$ which is used to calculate the statistically independent signal components $\hat{\mathbf{S}}$ using

$$
\hat{\mathbf{S}}=\mathbf{W X}
$$

where $\mathbf{X}$ is a matrix of the recorded multichannel signals. In this paper, we use the FastICA algorithm to determine the unmixing matrix, W [14]. Once the estimates of the original sources $\hat{\mathbf{S}}$ have been determined, the sources deemed to be artifacts can be removed by setting them to zero. The remaining sources are assumed to be the desired signal sources and when passed through the inverse of the unmixing matrix $\mathbf{W}^{-1}$ the resulting outputs are the artifact corrected signals [17].

As previously stated, ICA operates using multichannel recordings. However, using the proposed methodology, only single-channel signals are recorded for both fNIRS and EEG. A technique must, therefore, be implemented to generate a multichannel signal from a single-channel recording to allow for the use of the ICA algorithm. In this paper, the multichannel signal was generated using a method known as EEMD.

Empirical mode decomposition (EMD) is a technique, first described in 1998 [18], for nonlinear signal processing and is well suited for nonstationary signals. The method operates by decomposing a signal into individual components called intrinsic mode functions (IMFs) which have well-defined instantaneous frequencies. Once the IMFs have been determined, they can be employed as the required multichannel input to the ICA algorithm.

IMFs determined using the EMD algorithm are known to sometimes have problems due to mode mixing. Mode mixing can lead to serious aliasing in the time-frequency distribution and also make physical meaning of individual IMF unclear [19]. Ensemble EMD, as described in [20] is an extension to the 

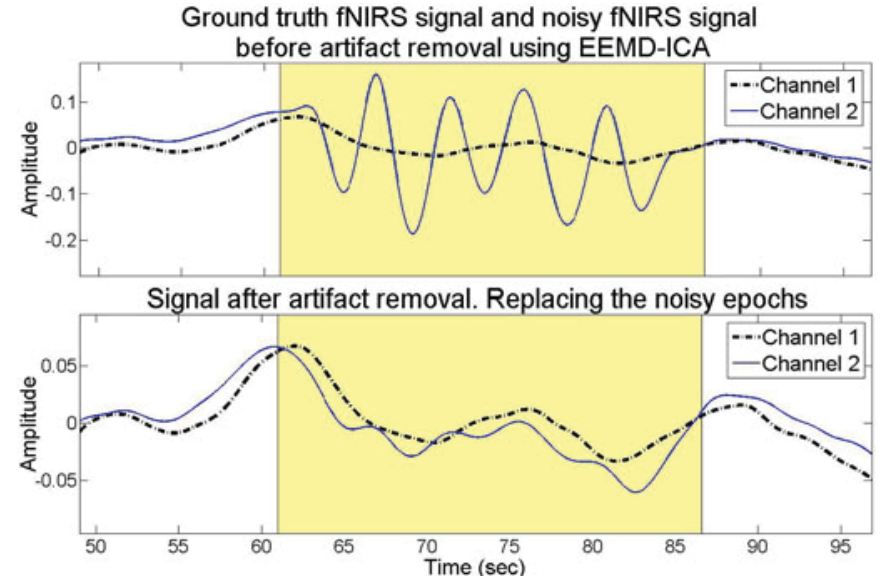

Fig. 9. Example of the output of the EEMD-ICA algorithm using the fNIRS data.

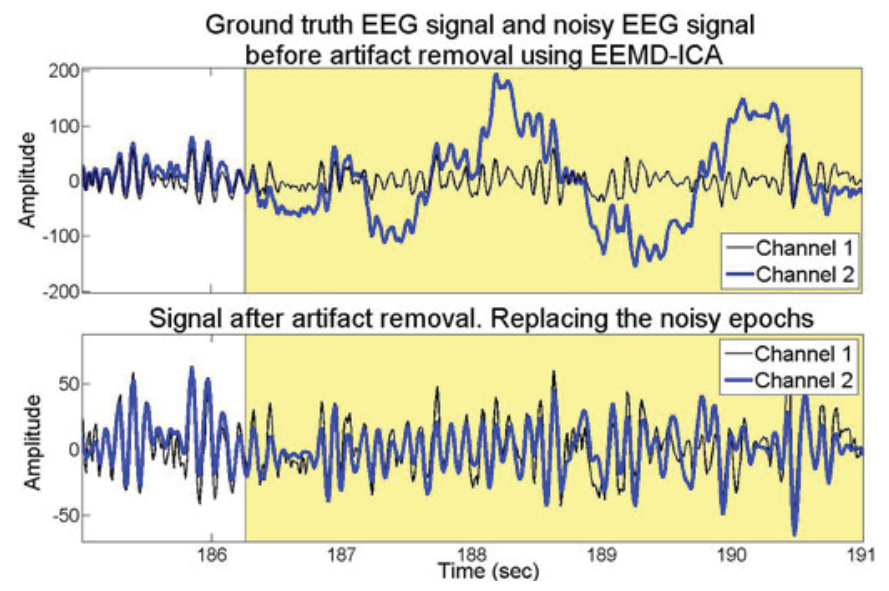

Fig. 10. Example of the output of the EEMD-ICA algorithm using the EEG data.

EMD algorithm which eliminates this problem. EMD-ICA and EEMD-ICA have been previously employed by researchers to remove different artifacts including eye blinks [21] and ECG artifacts from EEG [22].

The EEMD algorithm was applied to the artifact contaminated single-channel recordings from all trials for both fNIRS and EEG. An ensemble number of one hundred was used to avoid mode mixing in the IMF signals. Following the calculation of the signal IMFs, the FastICA algorithm was employed. The resulting independent components (IC's) were then manually analyzed and the components identified as artifacts were removed. The artifact components were manually determined using properties such as shape, frequency, and amplitude. The IMFs were reconstructed using the ICA unmixing matrix $\mathbf{W}^{-1}$ and the original single-channel signal minus the determined artifact components was then regenerated by summing the artifact free IMF. For example, outputs from the EEMD-ICA technique for both fNIRS and EEG are shown in Figs. 9 and 10, respectively.

Again, the EEMD-ICA technique was shown to improve both the correlation and the SNR values of Channel 2 with respect to Channel 1 . The average correlation increased by $32.46 \%$ to
TABLE 1

COMPARISON OF THE RESULTS FOR BOTH THE EEG AND fNIRS EXPERIMENTS OBTAINED With THE SELECTED ARTIFACT REMOVAL TECHNIQUES: THE IMPROVEMENT IN CORRELATION AND THE CHANGE IN SNR ARE PRESENTED

\begin{tabular}{|l|c|c|c|}
\cline { 2 - 4 } \multicolumn{1}{c|}{} & Adaptive Filter & Kalman Filter & EEMD-ICA \\
\hline EEG - Corr Imp. \% & $37.66 \%$ & $83.13 \%$ & $76.5 \%$ \\
EEG - $\Delta$ SNR & $5.1 \mathrm{~dB}$ & $9.7 \mathrm{~dB}$ & $8.9 \mathrm{~dB}$ \\
\hline fNIRS - Corr Imp. \% & $68.22 \%$ & $66.55 \%$ & $32.5 \%$ \\
fNIRS - $\triangle$ SNR & $5.0 \mathrm{~dB}$ & $5.5 \mathrm{~dB}$ & $2.3 \mathrm{~dB}$ \\
\hline
\end{tabular}

0.64 for the fNIRS data and by $76.5 \%$ to 0.73 for the EEG data. The SNR also rose for both modalities by $2.34 \mathrm{~dB}$ for the fNIRS data and by $8.89 \mathrm{~dB}$ for the EEG data.

\section{DISCUSSION}

Motion artifacts are a serious problem in biomedical signal processing, since they can corrupt the integrity, and hence compromise the interpretation, of many physiological signal recordings obtained by various methodologies, e.g., fNIRS and EEG. New and improved algorithms are continually being developed to eliminate the noise and estimate the desired signal of interest. However, without the knowledge of the true signal, performance evaluation of the algorithms is limited at best.

In this paper, a novel experimental setup is proposed that allows the simultaneous collection of the recordings of both the true artifact-free signal and the motion artifact corrupted signal of either fNIRS or EEG for the first time. The proposed technique is a modified sensor setup that employs multiple sensors (light detectors for fNIRS and electrodes for EEG) to monitor similar measurement regions which will have highly correlated underlying biosignals. By applying controlled artifact to only one of the detectors (measured by independent accelerometers on both detectors), simultaneous measurement of corrupted and true/desired signal is possible. The utility of this unique setup has been shown for recordings of different neuroimaging modalities (fNIRS and EEG) and a selection of motion artifact removal algorithms from the literature (adaptive filtering, Kalman filtering, and EEMD-ICA algorithm). The results of this study suggest that the proposed setup enables a simple but effective means of acquiring an excellent estimate of the noise-free signal together with a motion artifact corrupted version. This dataset can then be successfully used in valid comparison of motion artifact removal algorithms in both fNIRS and EEG applications (see Table I) using metrics such as the SNR and correlation. Other performance metrics such as information content and frequency domain analysis could also be performed using this dataset for a more detailed evaluation of the techniques; however, this is beyond the scope of this paper. Such signal pairs are also of great utility for signal processing engineers seeking performance measures for newly developed filtering methods especially when seeking to compare relative effectiveness against other accepted techniques.

While the proposed method is restricted to the assessment of techniques for removing motion artifact it is precisely this sort of artifact that is proving such a bottleneck to effective home monitoring of outpatient health. A simple easily replicable methodology, as proposed here, should speed up the process 
by which new approaches to the artifact management problem can be investigated. The mentioned removal examples, used in this paper focused on artifact in a functional brain monitoring setting; however, the method can be readily applied to other physiological measurements at other sites on the body. An obvious and important extension is the examination of motion artifact during cardiac event monitoring. As physical exertion is often the cause of an arrhythmic event, motion artifacts can again often occlude the recording of the subtle, desired, cardiac arrhythmia signals.

A more comprehensive comparison and the evaluation of common artifact removal techniques for both fNIRS and EEG signals will be carried out in the future as a continuation of this study. The EEG and fNIRS datasets used for analysis in this paper and collected using the proposed methodology have been made available on PhysioNet [23].

\section{REFERENCES}

[1] S. Bunce, M. Izzetoglu, K. Izzetoglu, B. Onaral, and K. Pourrezaei, "Functional near-infrared spectroscopy," IEEE Eng. Med. Biol. Mag., vol. 25, no. 4, pp. 54-62, Jul./Aug. 2006.

[2] J. T. Gwin, K. Gramann, S. Makeig, and D. P. Ferris, "Removal of movement artifact from high-density EEG recorded during walking and running," J. Neurophysiol., vol. 103, no. 6, pp. 3526-3534, 2010.

[3] K. Sweeney, T. Ward, and S. McLoone, "Artifact removal in physiological signals-Practices and possibilities," IEEE Trans. Inf. Technol. Biomed., vol. 16, no. 3, pp. 488-500, May 2012.

[4] K. Sweeney, H. Ayaz, M. M. S. Ward, T. E. Izzetoglu, and B. Onoral, "A methodology for validating artifact removal techniques for fNIRS," in Proc. Ann. Int. Conf. IEEE Eng. Med. Biol. Soc., 2010, 31-Sep. 4, 2011.

[5] H. Ayaz, P. Shewokis, A. Curtin, M. Izzetoglu, K. Izzetoglu, and B. Onaral, "Using mazesuite and functional near infrared spectroscopy to study learning in spatial navigation," J. Vis. Exp., vol. 56, 2011.

[6] K. Sweeney, D. Leamy, T. Ward, and S. McLoone, "Intelligent artifact classification for ambulatory physiological signals," in Proc. Ann. Int. Conf. IEEE Eng. Med. Biol. Soc., Aug 31, 2010-Sep. 4, 2010, pp. 63496352.

[7] R. Oostenveld and P. Praamstra, "The five percent electrode system for high-resolution EEG and ERP measurements," Clinical Neurophysiol., vol. 112, no. 4, pp. 713-719, Apr. 2001.

[8] R. Chai, G. P. Hunter, S. H. Ling, and H. T. Nguyen, "Real-time microcontroller based brain computer interface for mental task classifications using wireless EEG signals from two channels," in Proc. 7th IASTED Int. Conf., Feb. 2012.

[9] D. G. Manolakis, V. K. Ingle, and S. M. Kogon, Statistical and Adaptive Signal Processing. New York: McGraw-Hill Science/Engineering/ Math, 1999.

[10] S. Haykin, Adaptive Filter Theory. NJ: Prentice Hall, 2002.

[11] M. Izzetoglu, P. Chitrapu, S. Bunce, and B. Onaral, "Motion artifact cancellation in NIR spectroscopy using discrete Kalman filtering," Biomed. Eng. Online, vol. 9, no. 1, pp. 16-31, 2010.

[12] R. Sameni, M. Shamsollahi, C. Jutten, and G. Clifford, "A nonlinear Bayesian filtering framework for ECG denoising," IEEE Trans. Bio. Eng., vol. 54, no. 12, pp. 2172-2185, 2007.

[13] G. Welch and G. Bishop, "An introduction to the Kalman filter," Tech. Rep., 2006.

[14] A. Hyvrinen, J. Karhunen, and E. Oja, Indpendent Component Analysis, S. Haykin, Ed. New York, Wiley-Interscience, Jun. 2001

[15] S. Choi, A. Cichocki, H. min Park, and S. Young Lee, "Review blind source separation and independent component analysis: A review," Neural Inf. Process.-Letters Reviews, vol. 6, no. 1, pp. 1-57, 2004.

[16] C. J. James and C. W. Hesse, "Independent component analysis for biomedical signals," Physiol. Meas., vol. 26, pp. 15-39, 2005.

[17] M. Chawla, H. Verma, and V. Kumar, "Artifacts and noise removal in electrocardiograms using independent component analysis," Int. J. Cardiol., vol. 129, no. 2, pp. 278-281, 2008.

[18] N. E. Huang, Z. Shen, S. R. Long, M. C. Wu, H. H. Shih, Q. Zheng, N. C. Yen, C. C. Tung, and H. H. Liu, "The empirical mode decomposition and the Hilbert spectrum for nonlinear and non-stationary time series analysis," in Proc. Royal Soc. London. Series A: Mathematical, Phys. Eng. Sci., vol. 454, no. 1971, pp. 903-995, Mar. 1998.

[19] Z. Shen, Q. Wang, Y. Shen, J. Jin, and Y. Lin, "Accent extraction of emotional speech based on modified ensemble empirical mode decomposition," in Proc. IEEE Instrum. Meas. Technol. Conf., May 2010, pp. 600 604.

[20] Z. Wu and N. E. Huang, "Ensemble empirical mode decomposition: A noise-assisted data analysis method," Adv. Adaptive Data Anal., vol. 1, no. 1, pp. 1-41, 2009.

[21] J. Lindsen and J. Bhattachatya, "Correction of blink artifacts using independent component analysis and empirical mode decomposition," Psychophysiology, vol. 47, no. 5, pp. 955-60, 2010.

[22] B. Mijović, M. De Vos, I. Gligorijevic and, J. Taelman, and S. Van Huffel, "Source separation from single-channel recordings by combining empirical-mode decomposition and independent component analysis," IEEE Trans. Biomed. Eng., vol. 57, no. 9, pp. 2188-2196, Sep. 2010.

[23] A. Goldberger, L. Amaral, L. Glass, J. Hausdorff, P. Ivanov, R. Mark, J. Mietus, G. Moody, C.-K. Peng, and H. Stanley, "PhysioBank, PhysioToolkit, and PhysioNet: Components of a new research resource for complex physiologic signals," Circulation, vol. 101, no. 23, pp. 215-220, Jun. 2000.

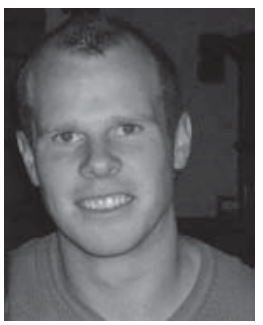

Kevin T. Sweeney (S'09) was born in County Meath, Ireland, in 1984. He received the B.E. degree (first class Hons.) in electronic engineering from the $\mathrm{Na}-$ tional University of Ireland, Maynooth, Ireland, in 2006, where he is currently working toward the Ph.D. degree in biomedical engineering.

His current research interests include biomedical signal processing specializing in the removal of motion artifacts from physiological signals.

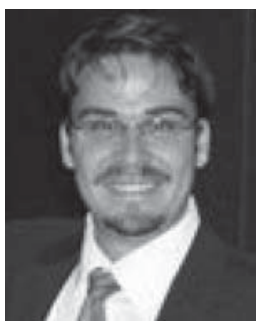

Hasan Ayaz (S'06-M'10) received the B.Sc. degree (Hons.) in electrical and electronics engineering from Boaziçi University, Istanbul, Turkey and the Ph.D. degree in biomedical engineering from Drexel University, Philadelphia, PA, in 2010.

$\mathrm{He}$ is currently an Assistant Research Professor at the School of Biomedical Engineering, Science and Health Systems, Drexel University, where he has developed enabling software for functional near infrared spectroscopy (fNIR)-based brain monitoring instruments. This technology was licensed by fNIR Devices LLC and Biopac Systems, Inc. He was also involved in the development of the InfraScanner, a portable-handheld medical device to detect hematoma in head trauma patients. His research interests include neuroengineering applications of human computer interaction and neuroergonomics, specifically, 1) development of noninvasive brain computer interfaces for communication and augmented interactivity in simulation and gaming settings and 2) optical brain imaging for assessment of cognitive workload and expertise development of operators such as such as air traffic controllers and unmanned aerial vehicle ground operators.

Dr. Ayaz is the recipient of the InfraScanner related awards which include Design of Medical Devices (DMD) Conference 2011 top presenter and Excellence in Design (EID) Gold Award.

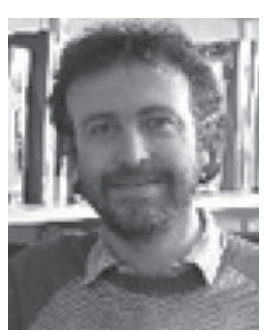

Tomás E. Ward (S'94-M'99-SM'12) received the B.E. degree in electronic, the M.Eng.Sc. degree in biomedical engineering, and the Ph.D. degree in biomedical engineering degrees from University College, Dublin, Ireland in 1994, 1996, and 1999, respectively.

In 1999, he was appointed as a Lecturer in the Department of Computer Science, National University of Ireland (NUI) Maynooth, Ireland, and in 2000, as a Lecturer in the Department of Electronic Engineering in the same university, where he is currently a Senior Lecturer. His current primary research areas include the application of brain computer interfaces for neurorehabilitation particularly in stroke, closed-loop communication protocols for distributed interactive applications, and virtual environments for cognitive and physical rehabilitation. 


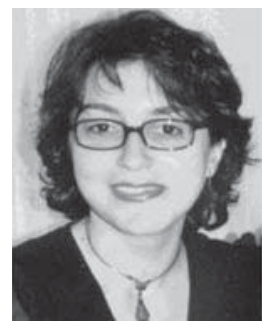

Meltem Izzetoglu received the B.S. and M.S. degrees both in electrical and electronics engineering from Middle East Technical University, Ankara, Turkey, in 1992 and 1995, respectively, and the Ph.D. degree in electrical and computer engineering from Drexel University, Philadelphia, PA, in 2002.

She is currently an Associate Research Professor in the School of Biomedical Engineering, Science and Health Systems at Drexel University. He teaches graduate and undergraduate level signals and systems, basic and advanced biomedical signal processing courses, and leads the signal processing effort in Optical Brain Imaging Group within Cognitive Neuroengineering and Quantitative Experimental Research Collaborative at Drexel University.

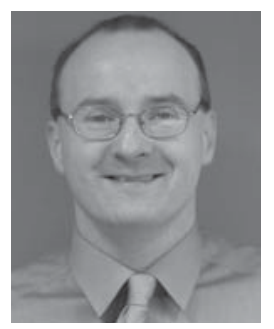

Seán F. McLoone (S'94-M'96-SM'02) received the Master of Engineering degree (with Distinction) in electrical and electronic engineering at Queens University Belfast, Belfast, U.K., in 1992, and the Ph.D. degree in control engineering in 1996.

He is currently a Senior Lecturer and the Head of the Department of Electronic Engineering, National University of Ireland, Maynooth, Ireland. His research interests include the general area of databased modeling and analysis of dynamical systems. This encompasses techniques ranging from classical system identification, fault diagnosis, and statistical process control to modern artificial intelligence inspired adaptive learning algorithms and optimization techniques. Current research activities include data analysis and signal processing for in-home health monitoring and assisted living technologies, algorithms for unsupervised sparse feature selection and clustering with application to large datasets.

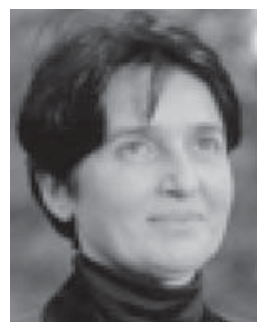

Banu Onoral (S'76-M'78-SM'89-F'93) received the B.S.E.E. and M.S.E.E. degrees from Boazii University, Istanbul, Turkey, and the Ph.D. degree from the University of Pennsylvania, Philadelphia, in 1978.

Her academic focus both in research and teaching is centered on biomedical signals and systems engineering. She has been a Founding Member of the Biomedical Information Technology Laboratory, Scaling Signals, and Systems Laboratory and the BioElectrode Research Laboratory, Cognitive Neuroengineering and Quantitative Experimental Research Collaborative, Drexel University, Philadelphia, where she is currently the H. H. Sun Professor of biomedical and electrical engineering. She has lead several curriculum development initiatives including the undergraduate telecommunication and biomedical engineering programs.

Dr. Onoral professional services include Chair and membership on advisory boards and strategic planning bodies of several universities and funding agencies, including service on the National Science Foundation Engineering Advisory Board (1997-1999), and on the proposal review panels and study sections. Her editorial responsibilities have included service on the Editorial Board of journals and the CRC Biomedical Engineering Handbook as a Section Editor for Biomedical Signal Analysis. She has been active in professional society leadership, in particular, national and international technical meeting organization. She served as the Vice-President for Conferences and as the President of the IEEE Engineering in Medicine and Biology. She also served on the inaugural Board of the American Institute for Medical and Biological Engineering. She has developed several signals and systems engineering software products and was recognized by the EDUCOM/NCRIPTAL Best Educational Tool Award. She is the recipient of a number of faculty excellence awards including the 1990 Lindback Distinguished Teaching Award of Drexel University. She is a Fellow of the IEEE Engineering in Medicine and Biology Society, Founding Fellow of the American Institute for Medical and Biological Engineering, Fellow of the American Association for the Advancement of Science, Senior Member of the Society of Women Engineers, and Member of the American Society for Engineering Education and the Scientific Research Society Sigma Xi. She is Listed in the Whos Who in the East, Whos Who in Science and Engineering, and Whos Who in the World. 